\title{
Event Driven Architecture for Travel Time Reduction
}

\author{
G.Nagappan \\ Associate Professor \\ Department of CSE \\ Saveetha Engineering College, Chennai, INDIA
}

\author{
Dr.C.Chellappan \\ Professor \\ Department of CSE \\ ANNA University, Chennai, INDIA
}

\begin{abstract}
To design an Event driven architecture for sensor-based networks, to analyse \& process complex event streams in Real Time urban traffic scenario. It is a multiagent system which is an aggregation of decomposed agents to communicate and coordinate with each other. This project aims to help every commuter to reduce his travel time by minimizing delay at traffic signals.
\end{abstract}

\section{General Terms}

Vehicular adhoc networks, Architecture

\section{Keywords}

Traffic control, Event Driven, Fuzzy Logic

\section{INTRODUCTION}

Across the nation, transportation representatives are sharing design concepts and producing transportation systems that will enhance the quality of life in our communities. Nationally, much attention and research is being conducted to help further the concept of Smart Transportation. In India, the urban development rate has increased five times over the rate of population increase.

Traffic in India has increased by over $60 \%$ while the population has remains growing too. Recognizing this fact, it is apparent that government will be unable to build its way out of congestion and will need to explore alternate solutions to "manage" congestion.

Smart Transportation considers future land use, road network, and flexible design standards to address transportation problems. Incorporating community goals such as improved safety and pedestrian accommodations and upfront identification of environmental constraints are key ingredients to any Smart Transportation solution.

Here a Multiagent system is formed using aggregation of decomposed agents to communicate and Co-ordinate with each other for the effective and efficient traffic control.

Table 1. Type of Agents

\begin{tabular}{|l|l|l|}
\hline \multicolumn{1}{|c|}{ Reactive } & \multicolumn{1}{c|}{ Cognitive } & \multicolumn{1}{c|}{ Hybrid } \\
\hline Make Decisions & Maintain Internal & Combination of \\
Runtime, based & representation of & both Reactive and \\
on simple & their world, the & Cognitive agent \\
Situation Action & mental state can & \\
rules & $\begin{array}{l}\text { be modified by } \\
\text { some symbolic } \\
\end{array}$ & \\
& reasoning & \\
\hline
\end{tabular}

Today one of the major problems faced by every city dweller is to reach the destination without getting struck due to the traffic. Most of them usually get irritated by waiting at the intersection of the road for the green signal.
But now this project aims to help every commuter to reduce his travel time by minimizing delay at traffic signals.

The Three major Issues to be handled by this project are that the traffic should be controlled by sensing the density of traffic on the road, The Allocation of signal timing should be based upon the density of traffic towards that direction and hence to provide more efficiency in management of traffic in the highly populated areas of the city.

\subsection{Objectives}

The objectives of the project are to Identify the density of the traffic by using sensor based networks.

To develop a dynamic traffic assignment system based on density of traffic and events.

Sending the updated traffic details at each and every point of time to the upstream intersection by using some technology.

To design an Event-driven architecture to analyze and process complex event streams in real time.

\section{ANALYSIS}

\subsection{Existing system}

The current traffic light controllers or message signs are based on fixed or ' Time-of-the-day' scheme using historical data. So there is no optimal control for the fluctuating traffic volumes.

The sudden changes in traffic due to planned or unplanned events like repair work, processions, accidents etc are not considered by the traffic light controllers.

\subsection{Proposed system}

In this paper we propose a new system in which action events based on Fuzzy Logic can be used for optimum control of fluctuating traffic volume even during over-saturated or unusual load conditions.

The Rules of fuzzy logic controller are formulated by following same protocols as a Human operator would use. The length of the current Green phase is extended or terminated depending upon 'arrival',

Number of vehicle approaching Green phase and 'Queue' , Number of vehicles in Red Phases.

\section{DESIGN}

\subsection{Event Driven Architecture}




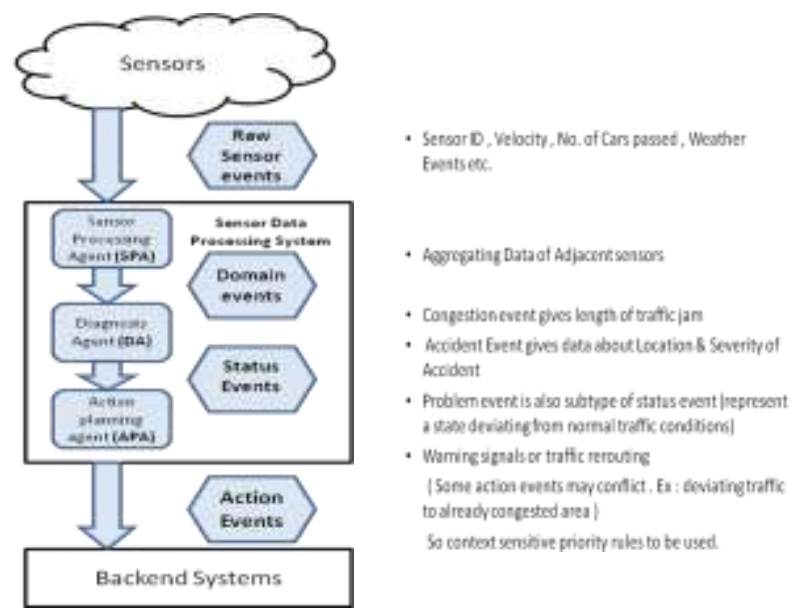

Fig.1. Event driven architecture
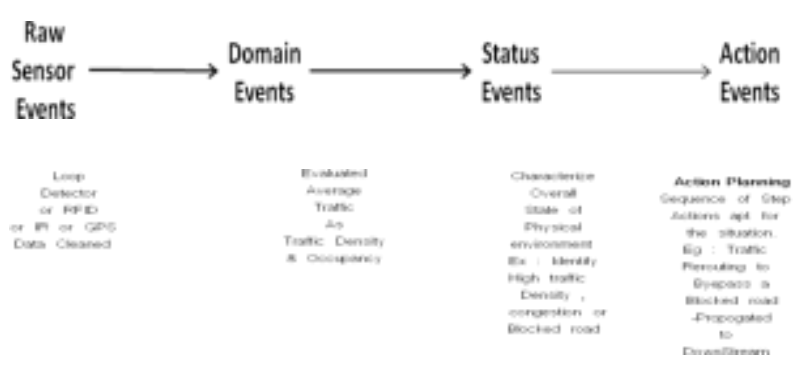

Fig. 2. Event Flow

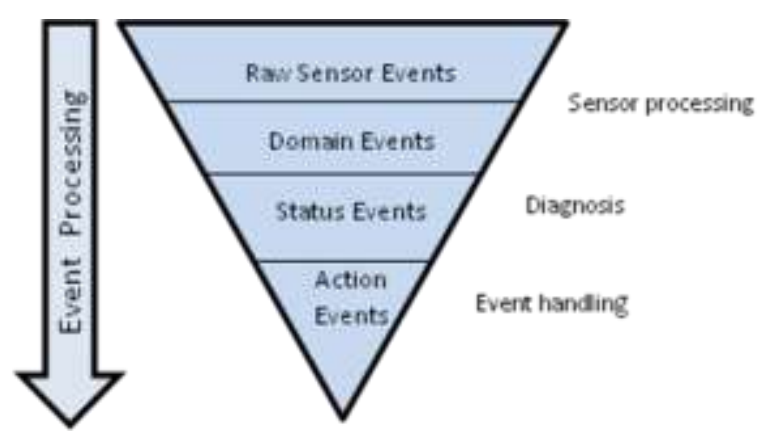

Fig. 3. Event Based Decision Support System

\subsection{Complex Event Processing Model}

The Steps of complex event processing are

\section{Step 1: Monitoring}

Each Event will have (Event ID \& Time stamp) and Event specific Information (Sensor ID \& Data)

\section{Step 2: Event Pattern Matching}

To Identify those pattern of events which signifies the meaningful state of the environment from a huge event cloud. (Use : Event processing Language )

\section{Step 3: Event Processing}

Filtering , Splitting, aggregating , transformation and enriching events. Even synthesizing new complex events from simple events. Ex: Accident $\rightarrow$ Traffic diversion. (Use : Rule engine for Execution )

\section{Step 4: Event Propagation}

Finally, events are propagated to downstream systems (Applications , Legacy systems or dash boards etc) for triggering appropriate event handling.

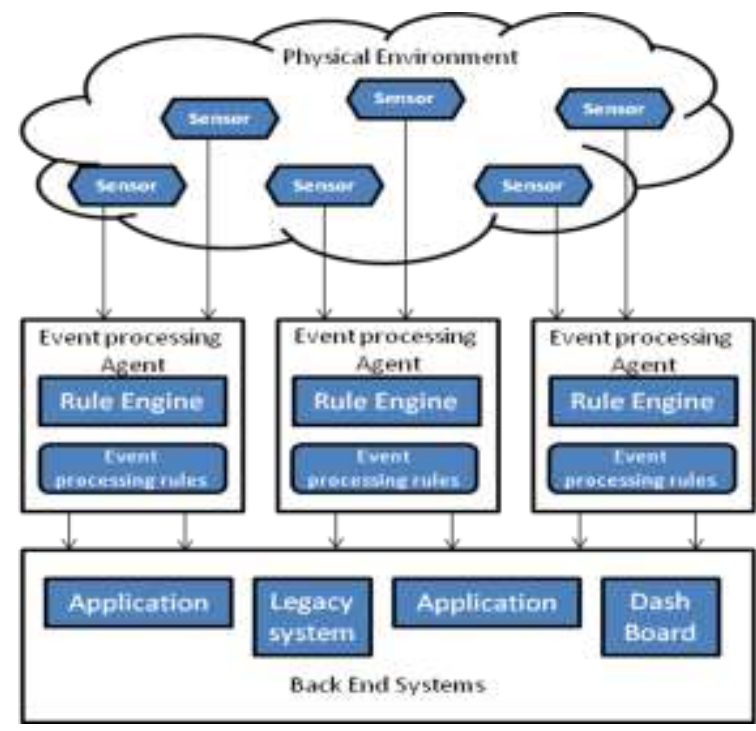

Fig.4. EDA Based Decision Support Systems

In high capacity road networks, sensors installed in the roads emit events when cars (Vehicles) are passing.

The Decision Support System transforms sensor events into more abstract \& sophisticated domain events for evaluating the actual traffic status (situation) \& initiates appropriate traffic control actions.

\subsection{Detailed Design}

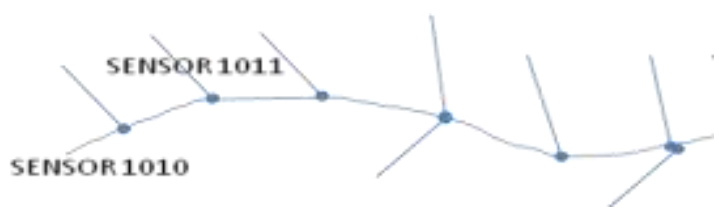

\section{Fig.5. EDA Based Decision Support Systems}

1. Traffic data registered by Detectors are received in the mobility management centre.

2. There decision is taken on what control actions to take to solve or minimize congestion

3. Actions include

a) Controlling the Traffic signal timings

b) Display messages on VMS (Variable message signals) panels installed above the road to warn drivers about traffic problems or recommend alternative routes

c) Contacting local authorities to send the right people to manage the situation 


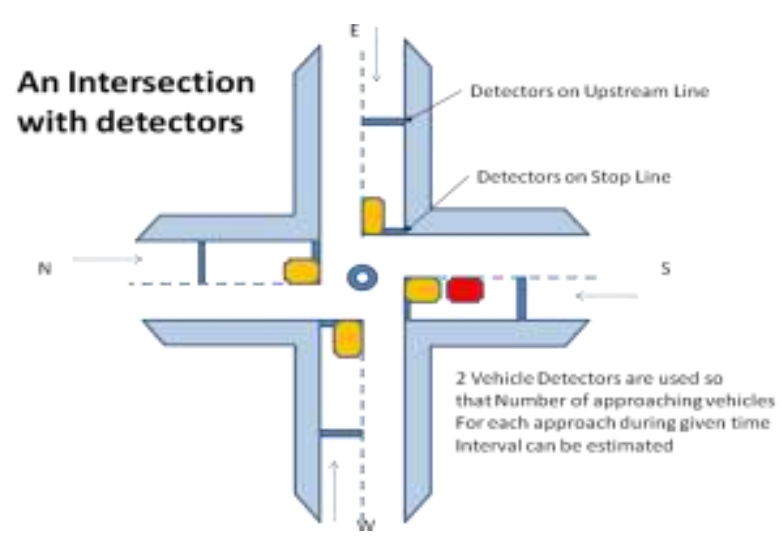

Fig 6. An Intersection with detectors

\subsection{Modeling of Multimodel Agent}

Aggregation of decomposed agents to communicate and co-ordinate with each other.

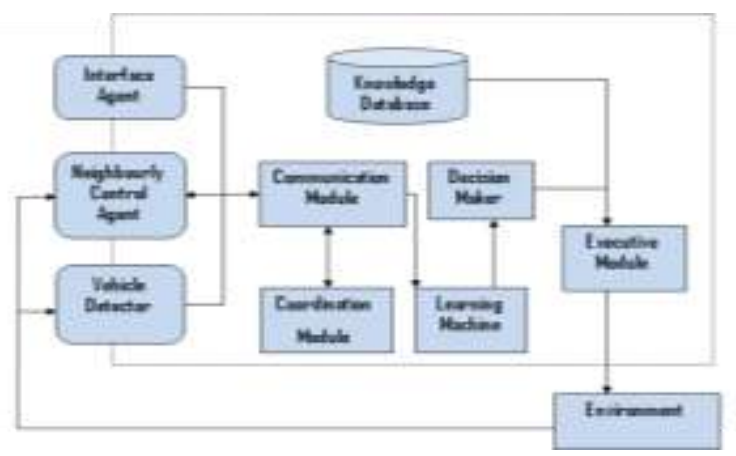

Figure 7. A Multiagent system

\section{IMPLEMENTATION \\ 4.1 Tool support}

- OWL inference engine + Editor $\rightarrow$ Structural event model ontologies

- $\quad$ Event processing language (EPL) + Processing engine $\rightarrow$ Event processing

(At present, no generally accepted standards exist for event definition, event pattern specification or rules languages \& engines )

- EPLs use ECA (Event Condition Rules) or SQL based approach for continuous queries over event streams

\subsection{Class Diagram}

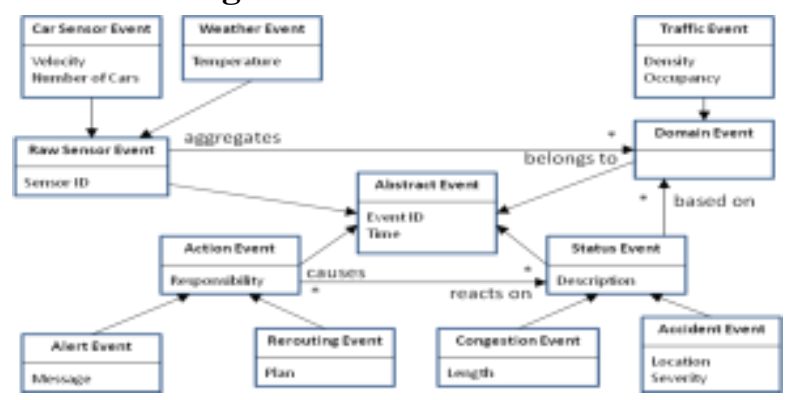

Figure 8. Class Diagram of Event Architecture

\subsection{Sample Aggregation Rule}

Insert into Section

Select 'ECRRoad' as id,

5640 as capacity,

avg(speed) as speed,

$\operatorname{avg}$ (occupancy) as occupancy,

$\operatorname{avg}$ (density) as density, sum(vehicles) as demand,

from Sensor.win:time(5 seconds)

where $\underline{\mathrm{id}}=$ ' 3014 ' or $\underline{\mathrm{id}}=$ ' 3049 ' or $\underline{\mathrm{id}}=$ '4231' or $\underline{\mathrm{id}}=$ ' 1234 '

output every 5 seconds

\section{Insert into Problem}

Select After.id as location,

'retention because of incident' as type,

After.demand - After.capacity as excess, 'problem' as category,

from Section.win:time(30 seconds) After, Section.win:time(30 seconds) Before,

where Before.nextSection $=$ After.id and

(Before.speed='LOW' or

Before.occupancy='HIGH') and

(After.speed='HIGH' or

After.occupancy='LOW')

Rules expressing expert (Traffic Constable) knowledge for local diagnosis \& action planning are represented in similar fashion in EQL rules for other Event Processing Agents.

\subsection{Fuzzy Variables}

- $\quad$ Arriving Variables (A)

- $\quad$ Queuing Variables (Q)

- Extension (EXT)

\begin{tabular}{|c|c|}
\hline Membership function(A) & $\begin{array}{l}\text { Queuing Vehicles(Q) at next } \\
\text { approach having red phase }\end{array}$ \\
\hline Few $\equiv-4$ to 4 & Few $=74$ to 4 \\
\hline Small $=0$ to 8 & $=0$ to 8 \\
\hline Medium $=4$ to 12 & Medium $=4$ ta 12 \\
\hline Many $=8$ to 16 & Many $=8$ to 16 \\
\hline
\end{tabular}

\section{Based on input fuzzy parameters, the duration of output} variable 'EXTENSION' is determined

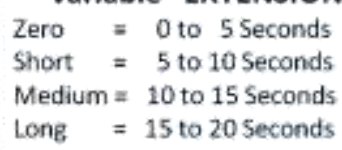

\subsection{Event Based Timing - Fuzzy Rule Base}

- For Extension or termination of current Green Phase

- The fuzzy controller after minimum Green time elapses, decides whether to extend after the time interval $\Delta \mathrm{t}$.

- Let us consider $\Delta \mathrm{t}=5 \mathrm{Sec}$ here.

- After a minimum green $(5 \mathrm{Sec})$

If Arrival is few AND Queue is (few OR small OR medium OR many) then Extension is zero else if Arrival is small AND Queue is (few OR small) then Extension is short

Then after first extension (ext1 + min green of $5 \mathrm{Sec})$ If arrival is few....

After second Extension, no extension shall be given to the current green phase. 
Next suitable green phase is selected based on the sequencing rule base, to maximize capacity of signalized intersection.

Weight, $w \mathrm{t}(\mathrm{A})$ of an approach is calculated from $\mathrm{Q}$ in each red phase.

$$
\begin{aligned}
& \text { Linguistic Labels for W(A) } \\
& \text { Zero } \quad(Z)=-4 \text { to } 4 \\
& \text { Low } \quad(\mathrm{L})=0 \text { to } 8 \\
& \text { Medium (M) } \quad=4 \text { to } 12 \\
& \text { High (H) } \quad 8 \text { to } 16
\end{aligned}
$$

The rules formulated gives priority to that approach with highest demand for green time.

\subsection{Fuzzy Rule Base ( From North )}

If Wait(W) is high then next green phase is $\mathrm{W}$ Else If Wait $(\mathrm{W})$ is medium and Wait $(\mathrm{S})$ and Wait(E) is low then next phase is $\mathrm{W}$

Else If Wait(W) and Wait(S) and Wait(E) is low then next phase is $\mathrm{W}$

Else If Wait(W) is less than high and less than high then next phase is $S$

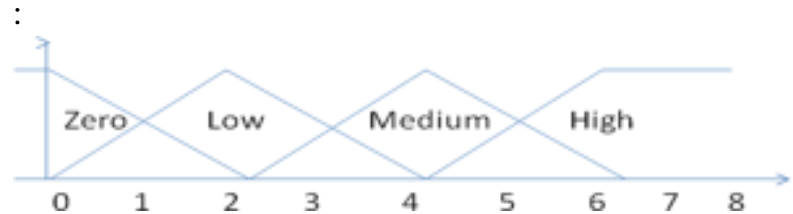

Fig 9. The density at various time intervals

\section{RESULTS AND DISCUSSION}

A prototype of the model was developed in embedded system using PIC 16F877 microcontroller.

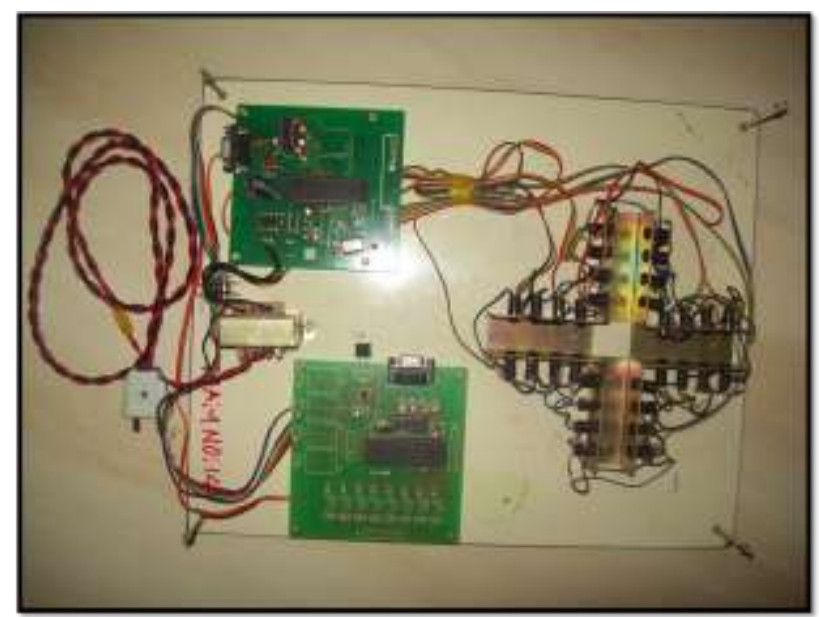

Fig 10. Prototype of the system

The prototype represents the traffic density on the lane at the instance of very little traffic. A message is sent to both forward and backward junctions of the road to control the up coming traffic.

The simulated scenarios of the traffic are as follows:

The Fig 11. represents the traffic density on the lane at the instance of very little traffic. The timer will be decreased in the current and next junctions of the road based on the fuzzy rules.

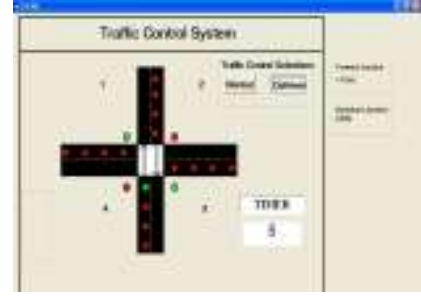

Fig 11. At very low traffic

The Fig 12. represents the traffic density on the lane at the instance of little traffic. The timer will be increased in the current, previous and next junctions of the road based on the fuzzy rules.

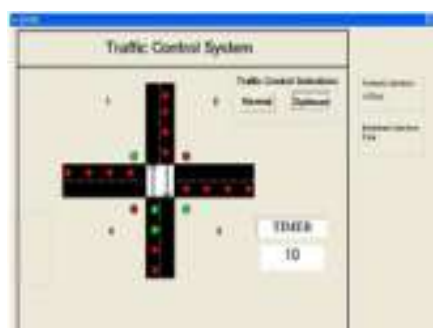

Fig 12. At low traffic

The Fig 13. represents the traffic density on the lane at the instance of medium traffic. The timer will be increased more than existing in the current, previous and next junctions of the road based on the fuzzy rules.

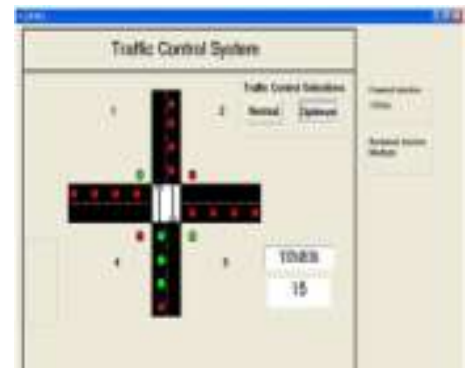

Fig 13. At high traffic

The Fig 14. represents the traffic density on the lane at the instance of very high traffic. The timer will be increased more than existing in the current, previous and next junctions of the road based on the fuzzy rules. Timer will also be considered for extension, based on traffic on other lanes.

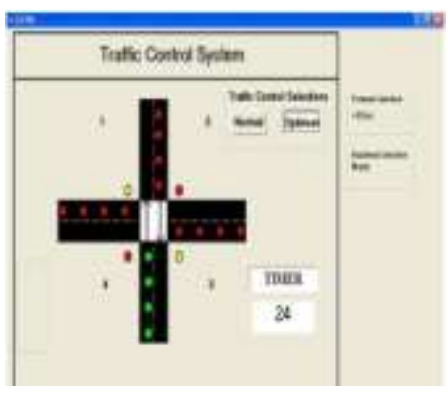

Fig 14. At very high traffic 


\subsection{Observations}

The Fig.15 represents the graphical representation of the vehicle density in each lane at particular instance. This graph shows the approximate number of vehicles present on each lane in $\mathrm{x}$-axis and the instance is specified in $\mathrm{y}$-axis.

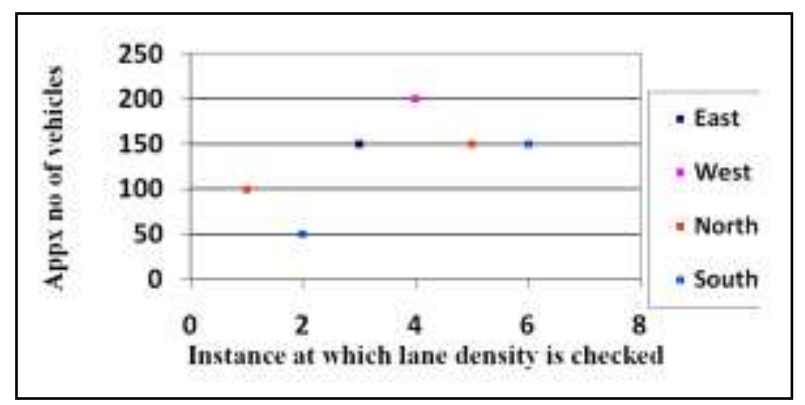

Fig 15. Graph of Density of Vehicles

The graph shows that the traffic is dynamically varying at different instances.

The Fig. 16 represents the average waiting time of the vehicles on various week days using manual ,average and event-based timing. This graph shows that our event based timing system reduces the average waiting time of the vehicles by huge factor than average timing and manual timing system which are being followed traditionally.

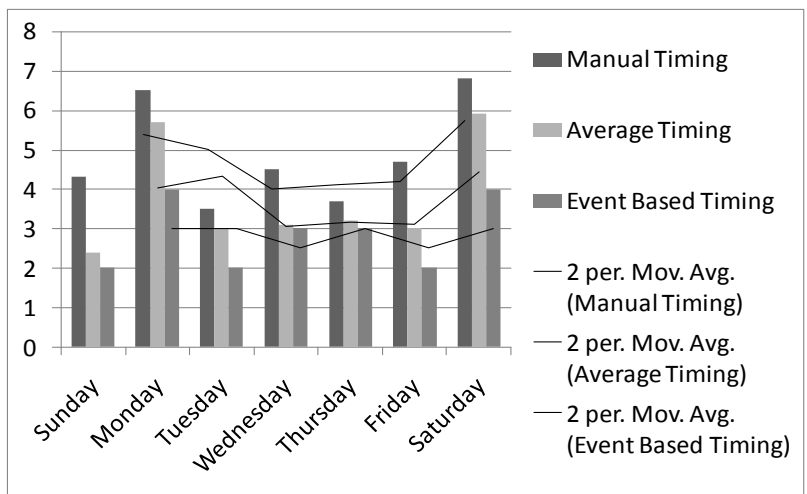

Fig 16. Average waiting time on weekdays

\section{CONCLUSION \& FUTURE WORKS}

Our proposed system dynamically adjusts the traffic signal timing autonomously, depending upon on the density of traffic and events. It also sends the updated traffic details at each and every point of time to the upstream intersection by using GSM technology. This event based timing system works well on both the light-traffic and heavily jammed traffic conditions.

The Average signal timing system reduces the waiting time of the vehicles at intersections when compared with Manual timing at intersections. The simulated results have proved that our Event Based Timing system is still robust when compared with Average signal timing system. Real world data would be collected to validate the developed methodology based of the Event driven architecture.

\subsection{Future works}

There remains a set of shortcoming in this work like

1. The Process must be repeated at short time intervals.

2. The Precise prediction of traffic of immediate future is not possible, because control action is based on optimizing current status only.

3. Detectors here cannot capture details of prevailing condition on the approach.

These issues can also be considered in a revised model of the prototype in future.

\section{REFERENCES}

[1] Chin Long Mak and Henry S. L. Fan, "Development of Dual-Station Automated Expressway Incident Detection Algorithms", IEEE Transactions On ITS, Vol. 8, No. 3, September 2007

[2] Aris Polychronopoulos, Manolis Tsogas, Angelos J. Amditis and Luisa Andreone, "Sensor Fusion for Predicting Vehicles' Path for Collision Avoidance Systems", IEEE Transactions on ITS, Vol. 8, No. 3, September 2007

[3] Wu, E., Diao, Y., Rizvi, S., "High-performance complex event processing over streams." Proceedings of the 2006 ACM SIGMOD Intern. Conf. on Management of Data, 2006, pp. 407-418.

[4] Clymer, J. R., Corey, P. D., and J. Gardner, "Discrete Event Fuzzy Airport Control", IN IEEE Transactions on Systems, Man, and Cybernetics, Volume 22, Number 2, March-April 1992, pages 343-351. 